\title{
Coronavirus COVID-19 - Theoretical and Practical Substantiations for Reducing Mortality from Complications
}

\author{
Albert Ivanovich Krashenyuk* \\ Krashenyuk A.I. Academy of Hirudotherapy, St. Petersburg, Russia \\ *Corresponding Author: Albert Ivanovich Krashenyuk, Krashenyuk A.I. \\ Academy of Hirudotherapy, St. Petersburg, Russia. \\ E-mail: Krashenyuk2013@yandex.ru
}

Received: April 15, 2020

Published: May 18, 2020

(C) All rights are reserved by Mohammed

Elbiaze.

\begin{abstract}
The high mortality rate and the enormous socio-economic consequences of the COVID-19 virus epidemic require the immediate development of effective treatment methods. The main complication of coronavirus infection, leading to the development of a fatal outcome, is pneumonia, respiratory and multiple organ failure, which develop in some patients with COVID-19 infection.

An analysis of the clinical, laboratory, and pathoanatomical picture of COVID-19 virus damage according to the literature suggests that there is a presence in the pathogenesis of patients with chronic disseminated intravascular coagulation (chronic DIC) and intraalveolar fibrinogenesis.

Prevention of these processes, early therapy using the systemic method of leech therapy (SMP) of hirudotherapy, which has about 30 types of therapeutic effects, including anticoagulant and thrombolytic effects, can prevent the development of severe, lifethreatening complications.
\end{abstract}

Keywords: COVID-19; Microthromboses; Systemic Method of Leech Therapy (SMP); Hirudotherapy

\section{Introduction}

The outbreak of the epidemic caused by the COVID-19 virus showed a high mortality rate from this virus not only in China but also in other countries: USA, Italy, Spain, and France. The main complication of coronavirus infection, leading to death, is respiratory and multiple organ failures.

By the time of writing, COVID-19 was reported in 187 countries. In total, more than 5 million people have contracted the coronavirus worldwide. more than 1.9 million people. of them recovered and over 328,265 people died.

Thanks to the studies of Chinese pathologists the picture of COVID-19 virus looks like as follows: "Blood clots in the small vessels of all organs, not only the lungs but also the heart, liver, and kidneys" described Bin Cao, MD, of the National Clinical Research Center for Respiratory Diseases in Beijing [1].

Pneumonia and respiratory failure develop in a small proportion of patients with COVID-19 infection. But they determine the catastrophic consequences of the epidemic.

Based on the publications of Chinese experts from Wuhan, one of the main markers of the development of fatal outcome is a high level of D-dimer and fibrinogen degradation products [1-6]. According to the observation of 191 patients with pneumonia, a D-dimer level of more than $1000 \mathrm{ng} / \mathrm{ml}$ upon admission to the hospital is associated with an 18-fold increase in the probability of patient death [3]. The level of D-dimer increased in patients with respiratory failure and was maximum in patients with fatal outcome [1,2]. In patients with a fatal outcome, the D-dimer index continuously increased in the dynamics of observation.

Laboratory and clinical signs of the development of Disseminated intravascular coagulation (DIC) were found in $71 \%$ of patients with pneumonia caused by the COVID-19 virus who were admitted to the hospital [5]. DIC-syndrome does not reach the stage of decompensation, culminating in the development of multiple microthromboses and Intra alveolar fibrinogenesis. Laboratory parameters in $71.4 \%$ of fatal patients met the criteria for disseminated intravascular coagulation, while at the same time, among survivors, similar shifts were observed in $0.6 \%$ of patients [4]. In another study, coagulopathy was observed in $50 \%$ of patients who died, and in $7 \%$ of survivors [6].

The clinical manifestations of damage to the heart, liver, kidneys, central nervous system in most patients with pneumonia caused by the COVID virus were non-specific and corresponded to secondary hypoxic changes characteristic of the development of respiratory failure [2,3,5-7].

Shifts in coagulogram activity were moderate. A minority of patients showed hypercoagulation in terms of activated partial thromboplastin and prothrombin time, more often dic-coagulation was noted. Elongation of prothrombin time was noted in $30 \%$ [2] and $62 \%$ of patients [8]. $26 \%$ of patients had hyperfibrinogenemia. 
Platelet count in severe patients decreases to the lower level of reference values $\left(160 \times 10^{9} / \mathrm{L}\right)$, providing sufficient protection against hemorrhagic complications [3]. Given the involvement of platelets in antiviral protection, this is a natural process dynamic.

Thrombocytopenia (below $100 \times 10^{9} / \mathrm{L}$ ) was observed in $5 \%$ of patients [3]. Among the clinical symptoms of the course of the viral infection of COVID-19, there are no signs of hemorrhagic complications $[2-4,8]$.

The therapy of DIC and venous thromboses developed by Z.S. Barkagan and his associates is based on anticoagulant and antiplatelet therapy [9].

There is a high probability of reducing mortality and cases of respiratory failure in patients with pneumonia caused by the COVID-19 virus when using anticoagulant and thrombolytic therapy. A decrease in the number of patients with pulmonary fibrosis and respiratory failure is expected after recovery from 2019-nCoV pneumonia.

Chinese experts used prophylactic anticoagulant therapy with heparin (50 units per kg of bolus weight and continuous infusion) in extremely severe patients with pneumonia caused by the $\mathrm{CO}$ VID-19 virus to provide membrane oxygenation of the blood and noted a positive effect [7]. However, clinics in Wuhan used heparin in a limited number of patients (9\%), primarily in patients with viral hepatitis $\mathrm{C}$ and with respiratory failure of the third degree [1, $2,7]$. As a result, it is not yet possible to assess the effectiveness of the use of anticoagulants according to Chinese experts.

Antiplatelet agents were used in one study [8]. According to observations of 12 patients with pneumonia caused by the COVID-19 virus, a positive effect of antiplatelet prophylactic therapy with dipyridamole (150 mg per day) was noted. Along with positive clinical dynamics in 11 patients, the authors noted a moderate slowdown in the growth of the level of D-dimer in patients (smoothing effect). One of the patients has died. When evaluating in vitro, a moderate ability of dipyridamole to inhibit COVID-19 virus replication was detected. The authors note that the decrease in replication was weaker than with chloroquine.

Due to the dominance in the pathogenesis of pneumonia caused by COVID-19, intravascular coagulation, micro-thrombosis in the vessels of the lungs and intra-alveolar fibrinogenesis, the clinical and laboratory picture fit into the framework of the chronic DIC.

A comprehensive analysis, based on the clinical and pathological results of Chinese specialists, has allowed authors from two reputable research centers: the Center for the Prevention of Thrombosis, Novosibirsk; Research Institute of Therapy and Preventive Medicine - a branch of the Federal Research Center for Cytology and Genetics Institute of the Siberian Branch of the Russian Academy of Sciences (Russia) to propose the following concept of therapy to reduce mortality caused by COVID-19 [11]:
1. Preventive approach. Conducting early anticoagulant therapy to prevent the development of DIC, and to prevent distress syndrome. The dynamics of the development of the disease allows you to start conducting it before the development of pneumonia, already on the first and second days of the development of the disease.

2. The use of therapeutic doses of anticoagulants (unfractionated heparin and low molecular weight heparins) for preventive therapy.

3. The use of outpatient thrombolytic drug Thrombovazim for the prevention and treatment of distress syndrome, DIC. Its use is advisable in moderate and severe patients from the early stages of the disease, and patients at risk using standard doses of the drug (800 ME 2 times a day oral). With the development of pneumonia, it is possible to combine the use of Thrombovazim with the use of prophylactic doses of low molecular weight heparins.

4. The use of antiplatelet therapy (dipyridamole, aspirin) to prevent microcirculatory blood flow disorders in all patients.

5. Mandatory use of thrombolytic therapy with Thrombovazim or anticoagulants in all patients with pneumonia.

6. The use of thrombolytic drugs of recombinant tissue plasminogen activators in patients with multiple organ failure.

7. A marker of a high risk of adverse outcome should be considered a level of D-dimer of more than $1000 \mathrm{ng} / \mathrm{ml}$. Patients in this group should receive Thrombovazim therapy or anticoagulant therapy.

8. Identification of risk groups for possible hemorrhagic complications according to risk scales. In these patients, it is possible to refuse to use a combination of thrombolytic and anticoagulant therapy, limiting therapy to standard doses of drugs. With a decrease in platelet count to $140-160 \times 10^{9} / \mathrm{L}$, laboratory monitoring is possible using a hemolysate-aggregation test and a leukocyte-platelet test.

The practice of using thrombolytic drugs is limited by possible adverse reactions and the need for laboratory monitoring.

In this work [11], a distinct and specific approach to the treatment of severe complications caused by the COVID-19 virus is described. In world literature, this is perhaps the most coherent concept for the treatment of pneumonia caused by COVID-19. By the way, these recommendations became possible only after prompt informing the world medical community by Chinese specialists who received convincing evidence of the pathogenesis caused by this type of coronavirus.

Without reducing the significance of the proposed therapy, we propose an alternative way to solve the problem of reducing mortality from pneumonia and other complications caused by COVID-19. 
Earlier, we expressed this point of view in the treatment of elderly people with multiple organ pathologies, namely, they become primarily victims of this pneumonia [12].

This is the application of the systemic method of leech therapy (SMP) (hirudotherapy). The method is based on a combination of the principles of homeopathy and acupuncture (hirudopuncture, our definition). The proposed solution is based on the almost 30-year-old use of SMP in elderly people with multiple organ pathologies and children undergoing treatment at the Academy of Hirudotherapy of St. Petersburg [12].

We will consider the preventive and treatment plan for anticoagulant and antiplatelet therapy for the prevention of DIC and distress syndrome in stages from the perspective of the systemic method of leech therapy (SMP).

Early anticoagulant therapy: Absolutely effective method. We offer this therapy in the form of the appointment of a medical leech (ML) Hirudo medicinalis.

Professor of Royal College of Birmingham (England) John Highcraft (J.B. Haycraft (1884) was the first who managed to obtain an extract from the ML that exerts an anticoagulating effect in vivo and in vitro. Although J.B. Haycraft did not find glands that produce anticoagulants, he showed that they must be sought in front of the body of the ML. And this is his great merit and priority.

The name "hirudin" he received in 1904. as a result of the work of F. Franz, performed in 1903. in the laboratory of Y. Jacoby (cited in [13], p. 159]). The complete primary structure of hirudin was established in 1976 [14]. It was later confirmed [15] and in 1985. the location of disulfide bonds in the hirudin molecule was established [16].

In 1986, the first publications on the production of recombinant desulfated hirudin appeared [17]. Thus, it is obvious that hirudin is a unique thrombin inhibitor. It is the only inhibitor capable of blocking the activity of a proteolytic enzyme in a picomolar concentration. The unique specificity of hirudin of a medical leech is explained by a previously unknown mechanism of multiple interactions between an enzyme and an inhibitor.

Hirudin forms an exceptionally strong complex with thrombin and the molecular basis for such strength is the large number of contacts between thrombin and hirudin that are detected by X-ray diffraction analysis of the corresponding hirudin crystals with an inhibitor.

The format of this article allows us to make only the most general overview of the properties of hirudin. But these properties also allow us to judge its unique anticoagulant potential.

Studies in the field of recombinant hirudin production have led to the appearance of various structural variants of a natural inhibitor, common to which is the presence in the molecules of the sulfated Tyr-63 residue. which determined their common name " desulfogirudin “ or "desirudin” [18].

Unlike natural, recombinant hirudins have a lower affinity for thrombin, although their anticoagulant properties are the same as those of a natural inhibitor. Hirudin inhibits not only free thrombin in solution but also an enzyme associated with a fibrin clot [19].

The clinical aspects of hirudin are diverse, the only limitation is the use of high doses of hirudin, in which increased bleeding in patients is possible. The inhibition of thrombin activity is not the only function of hirudin. In his presence, the thrombin activation reaction of coagulation factors V, VIII, XIII slows down. Hirudin inhibits the reaction of platelet release and aggregation by inhibiting the binding of thrombin to blood plates. Hirudin causes dissociation of the thrombin complex with specific proteins, platelet receptors, as the affinity for hirudin is higher in thrombin than for high-affinity platelet receptors.

\section{Destabilase the complex of ML secretion}

Destabilase, endo - and Exo - $\varepsilon$ - $(\gamma$-Glu) - Lys isopeptidase was first discovered in the secretion of salivary glands in 1986. The enzyme carries out its fibrinolytic (thrombolytic) activity through the hydrolysis of isopeptide bonds formed upon stabilization of fibrin in the presence of factor XIII blood coagulation, complementing new ideas about the mechanism of fibrinolysis.

Considering the behavior of destabilase in aqueous and organic solvents, it was suggested that the compound is micellar in nature, i.e. The ability to aggregate destabilase into larger complexes is realized in the micelle arrangement, which changes its spatial orientation depending on the polarity of the solvent.

The experiments showed that destabilase has a powerful prophylactic antithrombotic effect, primarily due to the blocking of platelet aggregation (platelet-vascular hemostasis) by the lipid component of destabilize.

It is noteworthy that destabilize also acts when administered orally to animals. It is obvious that destabilase has such a spatial structure that provides it with protection from proteolytic hydrolysis in the digestive tract. That is, destabilase can form aggregates, which, due to the lipid component, can change their spatial configuration, i.e. has a certain "fluidity". This assumption is supported by the fact that destabilase exhibits its properties (hydrolysis of isopeptide bonds) both in an aqueous medium and in organic solvents.

When analyzing the influence of destabilase on blood coagulation parameters, it was shown that in its presence the thrombin time and the time of recalcification of blood plasma are significantly extended. It is natural to assume that such action is provided by hirudin and a kallikrein inhibitor of blood plasma, which were found in drugs of destabilase. These facts suggest that the destabilase complex is a natural liposome [20]. 
The composition of the "destabilase complex" (DC) includes destabilase and prostaglandin components, hirudin and a blood plasma kallikrein inhibitor (IR). The strength of this complex is evidenced by the fact that it is not possible to destroy it by known biochemistry methods suitable for the destruction of liposomes. This is possible only as a result of the destruction of the polypeptide chain of destabilase. In this case, the prostaglandin component of destabilase, hirudin, and a kallikrein inhibitor is released.

The prophylactic antithrombotic effect of destabilase is due to both the blockade of the internal mechanism of blood coagulation (inhibition of platelet adhesion and aggregation and plasma kallikrein activity) and hirudin antithrombin activity. DK is also active when administered orally at a protein concentration of $2 \mathrm{mg} / \mathrm{ml}$ [20].

Liposomal nature of DCs also provides an important physiological role for this complex as a universal thrombolytic agent: fast and deep due to the wave - ultrasound effect [21,22] penetration of DC through the cell membrane, attachment due to the lipid component of destabilase to the damaged part of the vascular wall and parietal thrombus, slow lysis of the fibrin clot due to the isopeptide activity of destabilase and obstruction to further thrombosis due to thrombin block, plasma kallikrein adhesion, and adhesion aggregation.

Thus, the natural liposome - DC is an agent that provides both prophylactic and thrombolytic (therapeutic) action, which is an extremely important point in the treatment of thrombosis of origin.

Pharmacological properties of recombinant hirudins (r-hirudins): Given the importance of leech therapy in the prevention of thrombosis, it is necessary to dwell in more detail on the clinical effects of hirudin, which have been most fully studied with recombinant hirudin (r-hirudin).

As shown by preclinical trials and clinical and pharmacological studies ([21], p. 427), r-hirudin has a wide potential for action. Its anticoagulant activity is as high as that of the native inhibitor, and it can block all known functions of thrombin.

We present data on the pharmacodynamics and pharmacokinetics of recombinant hirudin [19].

Pharmacodynamics:

- $\quad$ Strong anticoagulant. It does not require endogenous cofactors.

- Specific thrombin enzyme blocker.

- It does not affect blood cells (platelets), plasma proteins and enzymes.

- In doses that inhibit thrombosis, it does not cause bleeding.

- Pharmacologically inert. Weak immunogen.
Pharmacokinetics:

- It is distributed extracellularly. Dose-dependent time half-life.

- High bioactivity after subcutaneous administration.

- Weak endogenous modulator. It does not metabolize in the liver.

- It is excreted in the urine in an active (unchanged) form.

- Not delayed in organs.

- Qualitative control and monitoring of therapy by measuring thrombin time or partial thromboplastin time, as well as other tests culminating in fibrinogen coagulation.

R-hirudin is fundamentally different from indirect anticoagulants since it does not affect the biosynthesis of proteins of the prothrombin complex, since coumarin, pelentan, warfarin, phenyline, etc.

It is a new type of anticoagulant and is used in the area in which heparin has so far been the only anticoagulant.

Therefore, numerous experimental and clinical work was carried out (treatment of acute coronary syndrome, prevention, and treatment of deep vein thrombosis, etc.) on the comparative effectiveness of r-hirudin and heparin.

The following advantages of r-hirudin over heparin were revealed:

- Its action does not depend on any cofactors, in particular, on antithrombin III, which is a cofactor of heparin. Therefore, hirudin can be prescribed to patients with antithrombin III deficiency;

- Hirudin does not bind or inactivate platelet-derived anti heparin factors such as platelet factor 4 . It does not affect platelet function. It cannot inactivate directly or indirectly through immune complexes. Therefore, hirudin (or ML) should be used instead of heparin in cases where the patient is threatened with thrombocytopenia stimulated by heparin;

- It is known that, unlike free thrombin, the enzyme involved in the composition of the thrombus is not able to inactivate either antithrombin III or the antithrombin III - heparin complex. On the contrary, hirudin, due to its structural features and low molecular weight, can block thrombin as part of a fibrin clot. Moreover, it can, in the same way, prevent the growth of an already formed thrombus and inhibit thrombin released from the thrombus as a result of endogenous thrombolysis [19].

Clinical use of recombinant hirudins in comparison with heparin

In 2002, the journal Seminar of Thrombosis and Hemostasis, Volume 28, No. 5, summarized the effectiveness of r-hirudin in comparison with heparin. 
The effectiveness of desirudin in acute coronary syndrome (ACS)

In a double-blind study of 113 patients with a low risk of stable angina, subject to coronary balloon angioplasty (CBA). They were divided into two groups receiving within 24 hours either desirudin or heparin.

All patients, starting from the day of surgery, were also prescribed aspirin for 4 weeks. Desirudin was administered with a $20 \mathrm{mg}$ bolus, and subsequently as an infusion of $0,16 \mathrm{mg} / \mathrm{kg} / \mathrm{h}$. Heparin - 10,000 units intravenous bolus and $0.12 \mathrm{mg} / \mathrm{kg} / \mathrm{h}$ as an infusion.

The preparations were titrated based on the value of APTT (activated partial thromboplastin time) within 85 - 120s. Acute coronary artery occlusion leading to myocardial infarction and requiring surgical intervention was observed in $10.3 \%$ of patients treated with heparin, and only in $1.4 \%$ of patients treated with desirudin. The differences were not statistically significant, which was explained by the authors with a small number of observations.

The GUSTO IIb study (The Global Use of Strategies to Open Occluded Coronary Arteries IIb Investigators, 1996) compared the clinical effects of hirudin and heparin in treating 12,142 patients with the acute coronary syndrome.

A statistically significant advantage of hirudin compared with heparin concerning the incidence of myocardial infarction (MI) and patient death in the first 24 hours from the start of treatment was noted in the analysis of cases with or without deviation of the ST ECG segment.

The first pilot studies on the effectiveness of desirudin in unstable angina in the presence of aspirin and anti-ischemic therapy showed that it causes more stable hypo-coagulation than heparin [22]. Repeated angiography performed after $72-120$ hours showed that in patients receiving hirudin compared with patients receiving heparin, the lesion site of the vessel decreased in size (p $=0.08)$, the minimum dose of the lesion increased $(p=0.028)$.

In the TIMI 5 study [23]. 246 patients were prescribed treatment with a thrombolytic agent alteplase (TAP) and aspirin. Within 6 hours from the onset of symptoms, 84 patients were additionally prescribed heparin and 162 desirudin. During the six-week observation period, mortality rates. As well as recurrences of myocardial infarction were significantly lower in patients receiving desirudin.

The goal of the HELVETICA study [24] in Europe was to compare the effects of hirudin and heparin in patients undergoing coronary balloon angioplasty (CBA). Patients with first-occurring angina pectoris, progressive resting angina pectoris received heparin or hirudin treatment before CBA.

The primary outcomes of clinical outcomes, including MI and death, are in favor of the benefits of r-hirudin over heparin. How- ever, clinical reports suggest that the security window with r-hirudin narrower than previously thought. Especially when used with thrombolytics and acetylsalicylic acid [19].

Prevention and treatment of postoperative deep vein thrombosis (DVT)

The ability of hirudin to block the thrombin enzyme associated with a thrombus determines the feasibility of its use for thromboembolic complications with a relatively low concentration of inhibitor in the blood. It is believed that the effectiveness of hirudin is maximal when used before and after thrombogenic stimuli.

The effectiveness of the use of r-hirudin in the prevention of DVT was evaluated in more than a thousand orthopedic patients [25] with two subcutaneous doses of 10,15 , and $20 \mathrm{mg}$. The drug was prescribed immediately before surgery and then - within 8 to 10 days after surgery. DVT was recorded according to the results of the phlebographic examination. The results indicate that $r$-hirudin, prescribed in doses of 15 and $20 \mathrm{mg} 2$ times a day, provided reliable and effective prevention of DVT. Statistical processing of the material showed that the proximal deep vein thrombosis level at the two doses mentioned was 3,1 and 2,4\%, respectively, compared to $19,6 \%$ in the group of patients who received heparin. The advantage of hirudin over heparin in the prevention of DVT during orthopedic operations was further confirmed by the same group of researchers [26].

Subcutaneous treatment was prescribed to 1000 patients. Twice subcutaneous administration over 9 days of 10,15 and 20 $\mathrm{mg}$ of hirudin was more effective than heparin, prescribed in 5000 units, 3 times a day. Using hirudin, a decrease in the risk of developing DVT was $30-48 \%$. The risk of developing proximal DVT decreased by $57-88 \%$.

According to [19], r-hirudin may be useful as a postoperative prophylactic factor. Based on its ability to inhibit clot-bound thrombin, it tends to prevent thrombolytic complications at relatively low systemic anticoagulation doses. DVT therapy was effective if it was started before thrombogenic stimulation or started immediately after it.

Recent data indicate that $r$-hirudin is more effective than unfractionated and low molecular weight heparins when used to prevent deep vein thrombosis in patients at high risk [27], including orthopedic patients [28].

In extracorporeal circulation and hemodialysis, heparin, often low molecular weight, is usually used as a blood preservative. However, in many patients, heparin causes the formation of antibodies that interact with platelets. And with endothelial cells, causing thrombocytopenia and thromboembolic complications. Therefore, patients with heparin-induced thrombocytopenia (GIT) require anticoagulant therapy [19] and the search for alternative anticoagulants to heparin is a serious problem for clinicians. 
The use of r-hirudin appears to be very promising in this regard, not only due to its anticoagulant properties but also due to its ability to be eliminated by the kidneys.

The use of an outpatient thrombolytic drug Thrombovazim for the prevention and treatment of distress syndrome, DIC. The properties of this drug that have been studied to date indicate its promise. But the hopes placed on him in combination with heparin are effectively resolved when using leech therapy.

The use of antiplatelet therapy (dipyridamole, aspirin) to prevent microcirculatory blood flow disorders in all patients is not required when using the systemic method of leech therapy.

The mandatory use of thrombolytic therapy with Thrombovazim or anticoagulants in all patients with pneumonia when using the systemic method of leech therapy is not required.

The use of thrombolytic recombinant tissue preparations plasminogen activators in patients with multiple organ failure using the systemic method of leech therapy is not required. Leech prostanoids have been found to inhibit platelet aggregation and stimulate the secretion of tissue plasminogen activator from the vascular wall. A marker of a high risk of an adverse outcome should be considered a level of D-dimer of more than $1000 \mathrm{ng} / \mathrm{ml}$. According to our long-term observations, the use of SMP allows reducing the level of D-dimer to the clinical norm.

Hemorrhagic complications during the long-term use of SMP were not detected (the observation period in some patients was more than 20 years of continuous use.

Over the past 25 years, a series of new fundamental facts have been obtained at the Academy of Hirudotherapy in St. Petersburg with the use of SMP, which can significantly enhance the clinical effect of its use and will reduce the mortality of patients with pneumonia caused by the COVID-19 virus.

Here is a list of the discovery of these new facts [29]:

- 1992 - The discovery of the phenomenon of "Hirudobiopsy" (A.I. Krashenyuk, S.V. Krashenyuk);

- 1993 - The discovery of the energy effect (informational entropy) (A.I. Krashenyuk,

- $\quad$ S.V. Krashenyuk);

- 1996 - The discovery of the neuro-stimulating effect of the medical leeches (A.I. Krashenyuk,

- $\quad$ S.V. Krashenyuk, N.I. Chalisova) [30];

- 2001 - The discovery of the acoustic effect of leech therapy (hirudotherapy). (A.I. Krashenyuk, D.I. Frolov);

- 2003 - The discovery of the detoxification effect of treatment (hirudotherapy). (A.I. Krashenyuk);
- 2004 - The discovery of the phenomenon of local hyperthermia in the body benign brain tumor - neurinoma (Kamynin Yu.F., Krashenyuk A.I.) [31];

- 2005 - The discovery of the negentropic effect of leech therapy (hirudotherapy).

- (A.I. Krashenyuk, G.N. Dulnev);

- 2007 - The role of autohemohirudotherapy in the formation of the immunomodulatory effect of leech (hirudotherapy). (A.I. Krashenyuk, S.V. Krashenyuk, A. Krylov);

- 2010 - The discovery of the hydro-structural effect of leech therapy (hirudotherapy).

- (Semikhina L.P., Krashenyuk A.I.).

\section{Conclusion}

The rapid increase in the number of publications allows focus on leech treatment by the systemic method (SMP). Consider the fundamental advantages of SMP over the proposed treatment methods for COVID-19.

Chinese scientists from the Beijing Institute of Biotechnology found that the anticoagulant heparin, which is used to treat a new coronavirus infection, causes deadly complications in patients with COVID-19. The research materials are published on the medRxiv preprint portal. BEIJING, May 1, 2020, 20:25 - REGNUM.

It is noted that doctors use the anticoagulant heparin when treating patients with a new coronavirus infection. Observations showed that this improved lung function by dissolving blood clots and reducing inflammation. However, heparin can spontaneously cause acute platelet deficiency, which leads to the formation of new blood clots and often leads to the death of patients.

Researchers have stated that patients with COVID-19 produce antibodies that bind to heparin molecules. The resulting complex damages the platelet cells with which heparin interacts. This leads to mass death of platelets. Such complications occurred in $40 \%$ of patients with COVID-19, in whom the disease proceeded in a complex form. However, usually such complications occur in $1 \%$ of patients. If patients were connected to an "artificial kidney" apparatus to purify blood of toxins, then patients were guaranteed to die from arising platelet insufficiency.

Thus, scientists conclude, for blood thinning in patients with COVID-19, it is necessary to use other anticoagulants, but not heparin.

Another powerful argument for the use of SMP in the treatment of COVID-19 is the publication of scientists from the USA and Germany on the suppression of the reproduction of coronavirus by cell protease inhibitors.

For SARS-CoV-2 to enter cells, it is necessary that its surface glycoprotein $\mathrm{S}$ be cleaved by cellular proteases. One of these proteases 
is the transmembrane enzyme TMPRSS2. However, as shown by studies by German scientists on cell culture of human airway epithelium, in addition to TMPRSS2, the virus needs another cellular protease - furin.

Furin and TMPRSS2 introduce gaps in different regions of the S protein, and both gaps are critical to the ability of the virus to enter the cell. Knockdown of TMPRSS2 using antisense RNA prevented the propagation of SARS-CoV-2 cell culture. In addition, virus propagation was suppressed by two TMPRSS2 inhibitors (MI-432 and MI-1900) and aprotinin, a broad-spectrum serine protease inhibitor. Virus replication was also suppressed by a furin inhibitor known as MI-1851. Moreover, the simultaneous action of TMPRSS2 inhibitors together with MI-1851 gave a more pronounced antiviral effect than the effect of an equimolar amount of each of the investigated TMPRSS2 inhibitors without MI-1851.

Scientists believe that TMPRSS2 and furin are promising targets for the treatment of COVID-19, and the use of their inhibitors, individually or in combination, should be considered as a way to treat the disease. Source: Dorothea Bestle., et al. "TMPRSS2 and furin are both essential for proteolytic activation and spread of SARS-CoV-2 in human airway epithelial cells and provide promising drug targets". bioRxiv, April 15, 2020; DOI: 10.1101/2020.04.15.042085.

In connection with the publication of the mechanism of activation of the coronavirus COVID-19, readers should be reminded of proteases inhibitors of medical leeches.

Bdellins - are a group of polypeptides with a small molecular weight, among which Bdellins A with a molecular weight of $7 \mathrm{kDa}$ are distinguished (bdelastazine with a molecular weight of 6.3 $\mathrm{kDa}$ is most studied in this group) and Bdellins B with a molecular weight of $5 \mathrm{kDa}$. Numerous forms of bdellins A and B were isolated by equilibrium chromatography; they are labeled A1 to A6 and B1 to B6. Both are potent inhibitors of trypsin, plasmin, and acrosin sperm. They do not block the activity of chymotrypsin, tissue and plasma kallikreins, subtilisin. They were first discovered by H Fritz., et al. in 1969. A recombinant form of bdelastazine was obtained.

Hirustazin - belongs to the same family of antistasin serine protease inhibitors. Isolated in 1994 from extracts of medical leeches. The molecular weight of girustazin is $5.9 \mathrm{kDa}$. It inhibits tissue kallikrein (but not plasma), trypsin, chymotrypsin and cathepsin $\mathrm{G}$ neutrophils. The ability of girustazin to block tissue kallikrein is a very important property, since the latter catalyzes the release of highly active kinins. Kinins through specific receptors on target cells modulate a wide range of biological activities, including those involved in maintaining normal blood pressure. Hirustazin is also obtained in recombinant form.

LDTI (Leech Derived Tryptase Inhibitor) is a tryptase inhibitor obtained from an extract of medical leeches. Tryptase is the main component of the secretory cytoplasmic granules of mast cells and leads to the destruction of extracellular matrix proteins. The important role of tryptase in allergic and inflammatory reactions is known. As with many of the compounds already described, recombinant LDTI has been created.

LCI (Leech Carboxypeptidase Inhibitor) is a carboxypeptidase A inhibitor. It was isolated in 1998 and has two isoforms with molecular weights of 7.3 and $7.2 \mathrm{kDa}$. It is steady in a wide range of $\mathrm{pH}$ and temperatures. Since this inhibitor is part of the secretion of the salivary glands of a medical leech, it can be assumed that it can block the hydrolysis of kinins by metalloproteinases at the site of biting of the leech of the skin, thereby enhancing the kinin-induced increase in blood flow. Created recombinant LCI.

Eglins are low molecular weight proteins from medical leech extracts with molecular weights of 8.073 and $8.099 \mathrm{kDa}$ ("b" and "c" forms, respectively). They were first described in 1977 by U. Seemuller., et al. Inhibit the activity of alpha-chymotrypsin, mast cell chymase, subtilisin and neutrophil proteinases, elastase and cathepsin G.

They have high resistance to denaturation and heating. The inhibitory spectrum of eglin "C" allows us to consider it one of the most important anti-inflammatory agents. However, there are very serious reasons to believe that eglins, which are isolated from extracts of a medical leech, are not present in the secretion of its salivary glands, but are secreted by the gastric gland.

The discovery of the wave effects of leeches in the kilohertz range and in the microwave/ultrahigh frequency range [32,33] allows us to consider another possible aspect of the therapeutic and preventive effect of SMP. To date, it has been established that a significant part of medical personnel in different countries is infected with the coronavirus COVID-19.

In this case, the most probable version is that the medical personnel violated the antiepidemic regime. However, there is one more reason - this is the remote (distant) interaction of the aquasystems of a sick and healthy person. Namely, we are talking about the discovery of Academician V.P.Kaznacheev, L.P. Mikhailov, S.P. Shurin „The phenomenon of intercellular distant electromagnetic interactions in a system of two tissue cultures" (Number and priority date: No. 122, February 15, 1966). The authors have discovered a new way of transmitting biological information.

The essence of the discovery: a culture of normal living cells was grown in two vessels. One culture was infected with the influenza virus. Almost simultaneously, cells in another vessel, which were only in optical contact with infected cells (the vessels were in contact with the bottoms of quartz glass), reproduced a similar pathological process. When the quartz glass was replaced with the usual one, the situation changed: the cells infected with the virus died, and their neighbors shared normally and developed well.

The authors of the discovery believe that between two tissue cultures in the optical range there are electromagnetic interactions. 
They showed the conditions under which a mirror cytopathic effect develops and developed ways to increase the sensitivity of healthy cells to the perception of electromagnetic radiation from affected cells. Using quantometric equipment, it was found that the process of cell damage, in particular by viruses, is accompanied by the characteristic kinetics of intrinsic electromagnetic radiation in the frequency range of the visible and ultraviolet spectral regions.

The discovery revealed a new information channel in biological systems, helped to develop a new approach to assessing the role of quantum phenomena in the mechanisms of functioning of the genetic program of a cell and the processes of encoding information in specialized cellular systems, such as neurons.

The correctness of the experiments of the authors was confirmed by many laboratories, including abroad. Confirmation was also performed at our department on the model of ultraviolet irradiation of people with the definition of chaos and order criteria on the device of Academician G.N. Dulnev "Eniotron” [34].

From this point of view, the protection of health workers requires a different design of protective clothing for physicians, i.e. protection against electromagnetic radiation from the aquasystem of a patient with human coronavirus COVID-19. Leech wave emissions are significantly superior to viruses influenza and herpes serotype 2 (from literature data 800 and $440 \mathrm{~Hz}$, respectively).

We do not yet know the radiation frequency of the coronavirus COVID-19, but we assume that it is significantly lower than that of a medical leech. And on this is based our assumption about the preventive role of SMP in health workers in contact with infected COVID-19 virus.

The immunomodulating effects of medical leeches are not well understood. Analysis of the literature shows that this is a multifactorial process, while discussing the activation of macrophagy of immunity, complementary systems and other levels of immune system response when using leeches. For the first time we paid attention to the currently unexplored property of leecing - autogemogirudatorapia, and proposed this term to refer to a new therapeutic factor of leechole therapy. We are talking about subcutaneous microbleeding effects resulting from the process of blood sucking, Figure 2. As for the topic of out-of-touch therapy, many publications speak about its benefits. Extensive reviews on this topic are of interest.concerns the topic of out-of-the-subject therapy, and many publications talk about its benefits. Extensive reviews on this topic are of interest. In the case of the TOPIC under discussion, COVID-19 should pay attention to the work. Immunomodulating effect of GT, aimed at specific restructuring of regulatory subpopulations, has been detected lymphocytes.

Thus, in some patients whose blood had an increased initial amount of CD4 (T-helpers) and CD25 (T-regulators) of lymphocytes subpopulations these indicators decreased to physiological values. Simultaneously with the inhibition of CD4 and CD25 subpopulations, there was an increase in the number and functional

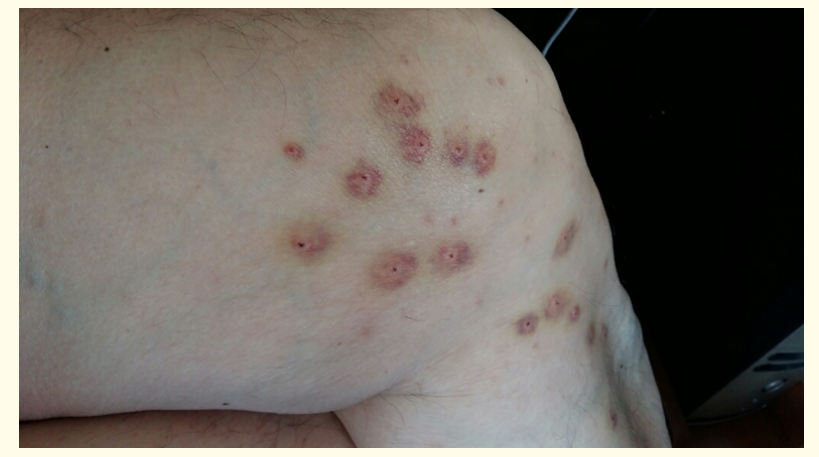

Figure 1: At the points of leech bite are visible subcutaneous hemorrhage. This is an illustration of autoimmunogirudotherapy., a phenomenon that has not been given to our research values in the assessment of the immunomodulating effect of leechole

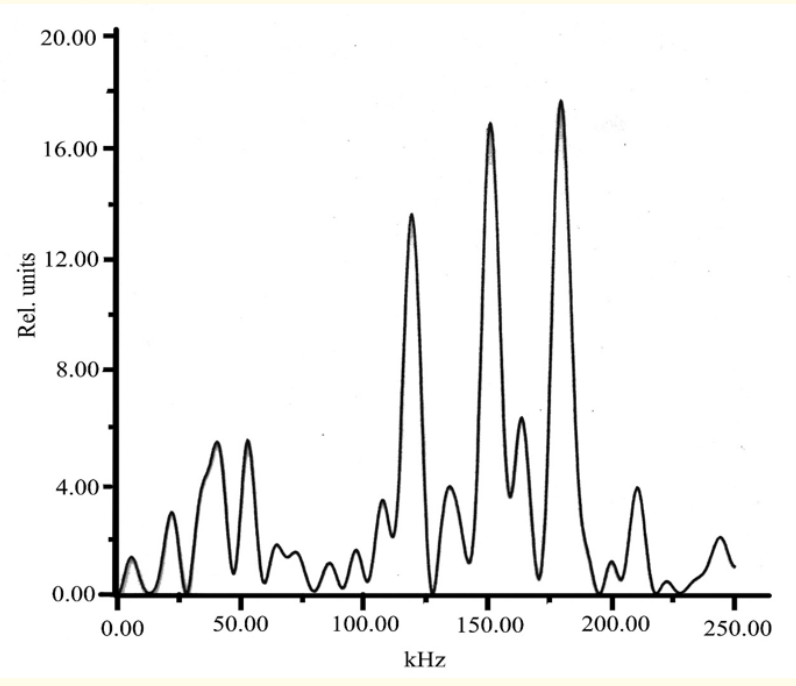

Figure 2: Recording an acoustic leech signal during the blood-sucking process after the Fourier conversion. The details of the experiment are outlined in the work.

indicators among SD8 (T-killers/suppressors) and SD16 (natural killers). In order to objectify the assessment of the helper-suppressor relationships of subpopulations in immunogenesis, the authors proposed a Regulatory Index (CD2 RI) in relation to the number of CD4 to the amount of CD8 and CD16, on the grounds that the latter subpopulations have common molecular-cell mechanisms of immunogenesis inhibition. Prior to GT, RI was 1.24 degrees 0.07 , which indicates the stress of the immune system and increased activity of CD4 (helper activation of immunogenesis). After GT, this figure changed to 0.86 to 0.07 due to the suppression of excessive activation of immunogenesis.

Thus, hirudotherapy induces the redestribution of immunocompetent cells in patient's body and modulates the helper/supressor mechanisms of immunogenesis at adequate homeostatic ranges.

A very important aspect of the problem under discussion is the fate of those who have recovered from COVID-19. The treatment 
tool is the presence in the leeway secret of enzymes collagenase and elastase, which affect connective tissue scars, causing their dissipation.

\section{Conclusion}

In conclusion of the proposed publication, I would like to express the hope that the materials presented in the publication will save many more human lives, as training in the NSR technique is available now doctors of any specialization at the Academy of Hirudotherapy.

When I published this article, I received the last issue of the journal JASM: John Weeks Editor-in-Chief reports that governments of India, China and other countries are actively promoting traditional methods of treatment and prevention against COVID-19.

The Government of India has issued a memorandum which indicates how potentially beneficial: yoga, Ayurveda, unani, siddha, homeopathy and and naturopathy. Note that leech therapy is one of the branches Ayurveda. The Chinese government attributes a relatively quick turnaround in this country towards the integration of traditional Chinese medicine with traditional biomedicine in $90 \%$ of their patients.

JACM Editor-in-Chief John Weeks issued the "Call to Action: Announcing the Traditional, Complementary and Integrative Health and Medicine COVID-19 Support Registry" to help launch the resource that was created by a global network of researchers. The registry is already backed by over a dozen practitioner organizations. While there remains no high-quality evidence to support integrative practices and natural agents against the virus, practitioners and consumers are experimenting with multiple natural health products and practices that existing evidence suggests might have preventive, supportive, complementary, or rehabilitative value.

\section{Bibliography}

1. ACC/Chinese Cardiovascular Association COVID-19 Webinar 1.

2. Chen N., et al. "Epidemiological and clinical characteristics of 99 cases of 2019 novel coronavirus pneumonia in Wuhan, China: a descriptive study". Lancet 395.10223 (2020): 507513.

3. Huang C., et al. "Clinical features of patients infected with 2019 novel coronavirus in Wuhan, China". Lancet 395.10223 (2020): 497-506.

4. Tang N., et al. "Abnormal coagulation parameters are associated with poor prognosis in patients with novel coronavirus pneumonia". Journal of Thrombosis and Haemostasis (2020).

5. Wu C., et al. "Risk Factors Associated with Acute Respiratory Distress Syndrome and Death in Patients with Coronavirus Disease 2019 Pneumonia in Wuhan, China". JAMA Internal Medicine (2020).
6. Zhou F., et al. "Clinical course and risk factors for mortality of adult inpatients with COVID-19 in Wuhan, China: a retrospective cohort study". Lancet 20 (2020): 30566-30563.

7. Guidelines for the prevention and treatment of new coronary viral infection COVID-19. The First Academic Clinic of Zhejiang University School of Medicine. Edition. Tingbo Liang. Moscow (2020): 92.

8. Liu X., et al. "Therapeutic effects of dipyridamole on COVID-19 patients with coagulation dysfunction". Preprint med Rxiv.

9. Barkagan ZS. “Hemorrhagic diseases and syndromes”. M: Medicine (1988): 236.

10. Lane DA., et al. "A novel user-friendly score (HAS-BLED) to assess 1-year risk of major bleeding in patients with atrial fibrillation: The Euro Heart Survey". Chest 138.5 (2010): 1093-100.

11. Gromov A.A., et al. "Coronavirus COVID-19- unapplied treatment possible" (2020).

12. Krashenyuk AI., et al. "The role of the systemic method of girulotherapy in the treatment of the elderly. Non-drug medicine". Scientific and Practical Journal 1 (2007): 59-73.

13. Baskova IP., et al. "Theory and practice". M (2004): 507.

14. Barabas E., et al. "Hirudin". Methods in Enzymology 45 (1976): 669-678.

15. Dodt J., et al. "The complete amino acid sequence of hirudin, a thrombin specific inhibitor". FEBS Letters 165 (1984):180183.

16. Dodt J., et al. "The complete amino acid sequence of hirudin. Localisation of disulfide bonds". Biological Chemistry 366 (1985): 379-385.

17. Harvey RP., et al. "Cloning and expression of cDNA coding for the anticoagulant hirudin from the blood sucking leech, Hirudo medicinalis". Proceedings of the National Academy of Sciences of the United States of America 83 (1986):1084-1088.

18. Matheson AJ and Goa KL. "Desirudin: a review of its use in the management thrombotic disorders". Drugs 60 (2000): 679700.

19. Markwardt F. "Hirudin as alternative anticoagulant - a historical review". Seminars in Thrombosis and Hemostasis 28.5 (2002): 405-414.

20. Nikonov GI and Latril J. "Scientific principles of hirudotherapy. In the book: Hirudotherapy and hirudopharmacotherapy". In the book: "Hirudotherapy and hirudopharmacotherapy (Edition. By Nikonov G.I.), 4 (2002): 28-38.

21. Krashenyuk AI and Frolov DI. "Interpretation of the nature of the energy-information effect of hirudotherapy based on an acoustic phenomenon. In the book: Science". Information. Consciousness., St. Petersburg (2001): 89-90.

22. Topol E.J., et al. "Recombinant hirudin for unstable angina pectoris. A multicenter, randomized angiographic trial". Circulation 89.4 (1994): 1557-1566. 
23. Cannon CP., et al. "A pilot trial of recombinant desulfatohirudin compared with heparin in conjunction with tissue-tipe plasminogen activator and aspirin for acute myocardial infarction: results of Thrombosis in Myocardial Infarction (TIMI) 5 trial”. Journal of the American College of Cardiology 23 (1994): 9931003.

24. Serruys PW., et al. "A comparison of hirudin with heparin in the prevention of restenosis after coronary angioplasty. Helvetica Investigators". New England Journal of Medicine 333.12 (1995): 757-763.

25. Eriksson BI., et al. "Direct thrombin inhibition with Rec-hirudin CGP 39393 as prophylaxis of thromboembolic complications after total hip replacement". Seminars in Thrombosis and Hemostasis 72.2 (1994): 227-231.

26. Eriksson BI., et al. "Prevention of deep-vein thrombosis after total hip replacement: direct thrombin inhibition with recombinant hirudin, CGP 39393". Lancet 347 (1996): 635-639.

27. Kemkes-Matthes B. "Hirudin for prophylaxis and treatment of deep vein thrombosis". Seminars in Thrombosis and Hemostasis 28 (2002): 455-458.

28. Nicolaides AN. "Clinical results with direct thrombin inhibitors". Pathophysiology of Haemostasis and Thrombosis 32.3 (2002): 36-38.

29. Dulnev GN and Krashenyuk AI. "From Synergetics to Information Medicine". St. Petersburg, Institute of Biosensory Psychology (2010): 167.

30. Krashenyuk AI. "Clinical Case of Conservative Leech Treatment of Neurinoma of the Brain". Acta Scientific Medical Sciences 4.1 (2020): 108-110.

31. Krashenyuk AI. "Nerotrophic (Neural Stimulating) and Neuromediator Effects of Hirudo medicinalis. Patrogenetic Mechanism of Treatment of Diseases of the Nervous System of the Human". Acta Scientific Medical Sciences 4.3 (2020): 01-06.

32. Krashenyuk AI., et al. "Wave effects of medical leeches. Bulletin of the "International medical leech center". Hirudotherapy and hirudopharmacotherapy. Edition. Doctor of Biological Sciences Nikonova G.I 4 (2002): 79-96.

33. Vlaskin SV., et al. "Resonance wave therapy and diagnostics. Study results and Prospects for use in leech therapy. Second World Congress hirudotherapy". Abstracts of Reports 7.8 (2016): 35-39.

34. Krashenyuk AI., et al. "Aqua-communication effect of ultraviolet irradiation and the phenomenon of the remote transmission of this effect from person to person". In the book: "Science. Information. Consciousness". Abstracts XIV. International Congress on Bioelectrography. St. Petersburg, 3.4 (2010): 27.

\section{Assets from publication with us}

- Prompt Acknowledgement after receiving the article

- Thorough Double blinded peer review

- Rapid Publication

- Issue of Publication Certificate

- High visibility of your Published work

Website: https://www.actascientific.com/

Submit Article: https://www.actascientific.com/submission.php Email us: editor@actascientific.com

Contact us: +919182824667 